\title{
The Incidence of Myocardial Diastolic Dysfunction in Patients with Decompensated Liver Disease
}

\author{
Hanan Zaghla, Emad Omer \\ Faculty of Medicine, Cairo University, Giza, Egypt \\ Email: hananza ghla@hotmail.com
}

How to cite this paper: Zaghla, $\mathrm{H}$. and Omer, E. (2019) The Incidence of Myocardial Diastolic Dysfunction in Patients with Decompensated Liver Disease. World Journal of Cardiovascular Diseases, 9, 404-418. https://doi.org/10.4236/wjcd.2019.96036

Received: May 14, 2019

Accepted: June 24, 2019

Published: June 27, 2019

Copyright $\odot 2019$ by author(s) and Scientific Research Publishing Inc. This work is licensed under the Creative Commons Attribution International License (CC BY 4.0).

http://creativecommons.org/licenses/by/4.0/

\begin{abstract}
Introduction: There is a debate about whether the occurrence is of systolic, diastolic dysfunction, or both in patients with liver cirrhosis. Aim of the work was to investigate the diastolic and systolic function changes prevalence in Acute decompensated on top of chronic liver disease. Patients and methods: The study was performed on three hundred patients with Hepatitis $C$ virus (HCV) associated liver cirrhosis; patients with a history of cardiac disease were excluded from the study about complete liver function tests. Abdominal ultrasound and echo-doppler were done for all patients and control. They were subdivided according to compensation into two groups: Group A was 150 patients with Compensated Liver Cirrhosis Disease (child A), and Group B was 150 patient with Decompensate Liver Cirrhosis (child B \& C) and Control group of twenty, with no hepatic abnormality and no cardiac dysfunction. Echocardiography was done to all patients to detect left ventricular end diastolic diameter (LVEDD), left ventricular end systolic diameter (LVESD), ejection fraction (EF\%) and $\mathrm{E} / \mathrm{A}$ ratio to detect the presence of diastolic dysfunction. Results: In this study, reversed E/A ratio as an indicator for diastolic dysfunction was found in 120 (40\%) patients while patients had standard E/A rate was $180(60 \%)$. E/A ratio decreased and decreased in the LVESD and EF\% in patients with decompensated liver cirrhosis more than those with compensated liver cirrhosis. Conclusion: Decompensated liver cirrhosis is associated with diastolic and systolic dysfunctions.
\end{abstract}

\section{Keywords}

Myocardial Dysfunction, Liver Cirrhosis, Hepatic Decompensation

\section{Introduction}

Patients with liver cirrhosis are reported to have a hyperdynamic circulation, 
which is manifested as increased cardiac output, decreased systemic vascular resistance, and systemic arterial vasodilatation, primarily [1].

The following clinical criteria generally defined the term CIrrhotic cardiomyopathy: baseline increased cardiac output but blunted ventricular response to stimuli, systolic and diastolic dysfunction, absence of overt left ventricular failure at rest, and electrophysiological abnormalities including prolonged QT interval on electrocardiography and chronotropic incompetence [1].

The impaired cardiac output may favor a decrease in renal perfusion, contributing to the pathogenesis of the hepatorenal syndrome. Patients with liver cirrhosis may suffer from various cardiac abnormalities, which may affect their outcome. Fatma et al. studied the diastolic dysfunction in cirrhotic patients through Tissue Doppler recording of the tricuspid and mitral annular diastolic velocities, but they worked on a small sample of patients [2].

There has been little published information regarding diastolic function in liver cirrhosis.

Strict diagnostic criteria for cirrhotic cardiomyopathy are deficient, and this syndrome is often not detected. No specific treatment or management strategies have been tested for patients with cirrhotic cardiomyopathy. The presence of the cirrhotic cardiomyopathy should be suspected in patients with worsening hemodynamics, and such patients may benefit from more meticulous monitoring and treatment of the underlying pathology leading to decompensation, and close monitoring during procedures suspected to cause decompensation (i.e. TIPS, paracentesis, and transplant). Clinical trials in this area are awaited. In the meantime, treatment of cirrhotic cardiomyopathy once identified, should follow the American College of Cardiology American Heart Association guidelines recommendations for the treatment of patients with heart failure [2].

Ventricular diastolic compliance and diastolic function can be assessed by measuring the velocity of blood flow from the left atrium to the left ventricle during early diastole (the $\mathrm{E}$ wave) and late diastole (the A wave) and calculating the E/A ratio by using the Doppler echocardiography [3].

\section{Aim of the Work}

This work aims to study the myocardial diastolic dysfunction by Doppler echocardiogram in patients with HCV related liver cirrhosis disease and compare compensated and decompensated liver diseases.

\section{Patients and Methods}

His study was carried on 300 patients and 20 controls done in the Internal Medicine Department of El Haram Hospital between February 2015 and March 2017. Patients were confirmed to have HCV related liver cirrhosis with clinical, laboratory and ultrasonography finding.

$\Rightarrow$ Group A: include 150 patients with compensated liver cirrhosis disease with hepatitis C.

$\Rightarrow$ Group B: include 150 patients with decompensated liver cirrhosis disease 
with hepatitis C.

$\Rightarrow$ Control group: 20 persons 11 (55\%) were females, and 9 (45\%) were males, the age ranged between 40 - 55 years with a mean age ( $48.8 \pm 3.27)$.

The following exclusion criteria were considered during patient selection:

- Age $>18$ years $<60$ years, Patients with hepatocellular carcinoma, Patients with cardiac disease as, Rheumatic heart disease. Congenital heart disease, Ischemic heart disease, Hypertensive heart disease. Cardiomyopathies, $\mathrm{Pa}$ tients with bilharzial cor pulmonary, Patients with severe anemia, Diabetes mellitus, Liver cirrhosis of any etiology other viral related Patients with HBV related liver cirrhosis.

\section{All patients and control groups were subjected to:}

1) Full history with particular emphasis to symptoms and signs of chronic liver disease, History of previous chronic illness that interfere with diastolic cardiac function (e.g. hypertension), The presence or absence of ascites, History of bilharziasis and History of viral hepatitis.

2) Clinical examination: A general examination and Local examination (abdominal and cardiac examination).

Laboratory investigations: Liver biochemical tests (Aspartate aminotransferase (AST), Alanine aminotransferase (ALT), Serum albumin, INR, Total bilirubin, and Hepatitis markers:

3) Abdominal ultrasonography: Ultrasonography examination was done in the fasting state; ultrasonographic study of the liver was done to assess its size and echogenicity to determine any pathology such as liver cirrhosis. The portal tracts, as well as the portal and splenic veins, were also measured. The rest of the abdomen was scanned for the presence of ascites or retroperitoneal masses. The spleen, gallbladder, and kidneys were also examined.

4) ECG.

5) Echocardiographic study: Two-dimensional echocardiography was performed to measure cardiac dimensions and diastolic functions (Left ventricular end diastolic diameter (LVEDD), left ventricular end systolic diameter (LVESD), left ventricular ejection fraction (LVEF), and E/A ratio (E, initial maximal ventricular filling velocity, $A$, late diastolic or atrial velocity)). The Doppler recording of the diastolic mitral flow was obtained in the apical four-chamber view by positioning the sample volume on the inflow area of the left ventricle, just as the level of the tips of mitral leaflets. Left ventricular outflow tract was measured by using the apical five-chamber view with the sample volume placed just below the level of the aortic leaflets. We calculate ejection fraction (EF), peak $\mathrm{E}$ (the early peak of the velocity of mitral inflow) and peak A (the late spike of mitral inflow), the ratio of $\mathrm{E} / \mathrm{A}$, information of study diastolic function.

\section{Statistical Analysis}

Data were collected, revised, coded and entered to the Statistical Package for Social Science (IBM SPSS) version 20. The data were presented as number and 
percentages for the qualitative data, standard deviations, mean and ranges for the quantitative data with parametric distribution and median with interquartile range (IQR) for the quantitative data with the nonparametric distribution.

Chi-square test was used in the comparison between two groups with qualitative data, and Fisher exact test was used instead of the Chi-square test when the expected count in any cell found less than 5 .

Independent t-test was used in the comparison between two groups with quantitative data and parametric distribution, and the Mann-Whitney test was used in the comparison between two groups with quantitative data and nonparametric distribution.

The confidence interval was set to $95 \%$, and the margin of error accepted was up to $5 \%$. So, the P-value was considered significant as the following:

- $\mathrm{P}>0.05$ : No significant (NS);

- $\mathrm{P}<0.05$ : Significant.

\section{Results}

The study was conducted on 300 patients with HCV related liver cirrhosis and 20 controls chosen randomly from the Internal Medicine and Department and intensive care of kasralainy Hospital between February 2015 and March 2017. They were subdivided according to compensation into three groups:

$\Rightarrow$ In group A: 150 patients with Compensated Liver cirrhosis disease (child A) $80(53.3 \%)$ females and $70(46.7 \%)$ males. The age ranged between $38-60$ years with a mean age (51.52 \pm 6.06$)$.

$\Rightarrow$ In group B: 150 patients with Decompensated Liver cirrhosis disease (child B \& C) $70(46.7 \%)$ females and $80(53.3 \%)$ males. The age ranged between 39 60 years with a mean age $(50.37 \pm 6.15)$.

$\Rightarrow$ In the control group: 20 persons 11 (55\%) were females, and $9(45 \%)$ were males, the age ranged between 40 - 55 years with a mean age $(48.8 \pm 3.27)$.

\section{A) Descriptive data of patients groups (A\&B)}

Descriptive data of patients group as regard laboratory, echocardiography \& age data (Table 1).

Table 1. Descriptive data of patients group revealed an increase in (AST-ALT-bilirubinINR-urea-creatinine) and decrease in (Albumin-Hb\%- EF\%) as regard laboratory \& echocardiography data (AST: aspartate transaminase, ALT: alanine transaminase, INR: international normalized ratio, NA: sodium, K: Potassium, HB\%: Haemoglobin).

\begin{tabular}{ccccc}
\hline & Min. & Max. & Mean & SD \\
\hline Age & 38 & 60 & 51.52 & 6.06 \\
AST & 20 & 111 & 52.00 & 20.02 \\
ALT & 24 & 110 & 61.78 & 18.83 \\
Albumin & 1.4 & 3.45 & 2.60 & 0.58 \\
INR & 1.2 & 3 & 1.90 & 0.35 \\
Bilirubin & 0.7 & 9.7 & 2.49 & 1.75 \\
\hline
\end{tabular}


Continued

\begin{tabular}{ccccc}
\hline Urea & 3.2 & 155 & 39.57 & 20.97 \\
Creatinine & 0.6 & 5 & 1.39 & 0.63 \\
$\mathrm{Na}$ & 125 & 145 & 135.95 & 4.37 \\
$\mathrm{~K}$ & 2.9 & 5.3 & 4.01 & 0.59 \\
$\mathrm{Hb} \%$ & 9.5 & 12.5 & 11.19 & 0.74 \\
Platelet & 147 & 325 & 203.75 & 42.98 \\
LVEDD & 3.5 & 6.2 & 5.16 & 0.55 \\
LVESD & 3.2 & 5.6 & 4.06 & 0.41 \\
EF\% & 45 & 70 & 59.08 & 4.07 \\
\hline
\end{tabular}

\section{B) Comparison between patients and control groups}

1) As regard sex distribution. There was no difference in sex distribution among the patient group (150 males and 150 females) while $55 \%$ of the control group were females ( $\mathrm{P}$-value $=0.698)$.

2) As regard to age distribution. The age of the involved patients ranged from 18 to 60 , with a mean of 51.27 , while the mean age of the control group was 48.8 $(\mathrm{P}$-value $=0.087)$.

3) Laboratory data patients group and control group; liver function test (ASTALT-Bilirubin-INR) were higher in patients group than control group with highly significant difference (P-value $<0.001$ ) while (Albumin-Platelet-Hb\%) were lower in patient groups than in the control group with highly significant difference $(\mathrm{P}$-value $<0.001)$ (Table 2).

Table 2. Laboratory findings in patients and control.

\begin{tabular}{ccccccc}
\hline & \multicolumn{2}{c}{$\begin{array}{c}\text { Patients group } \\
(\text { No. }=300)\end{array}$} & \multicolumn{2}{c}{$\begin{array}{c}\text { Control group } \\
(\text { No. }=20)\end{array}$} & \multicolumn{2}{c}{ Independent t-test } \\
\cline { 2 - 7 } & Mean & SD & Mean & SD & T & P-value \\
\hline AST & 52.00 & 20.02 & 28.40 & 4.69 & -5.202 & $<0.001$ \\
ALT & 61.78 & 18.83 & 32.10 & 5.24 & -6.930 & $<0.001$ \\
Albumin & 2.60 & 0.58 & 3.73 & 0.25 & 8.464 & $<0.001$ \\
INR & 1.90 & 0.35 & 1.14 & 0.26 & -9.018 & $<0.001$ \\
Bilirubin & 2.49 & 1.75 & 0.70 & 0.40 & -4.513 & $<0.001$ \\
Urea & 39.57 & 20.97 & 29.90 & 3.69 & -2.044 & 0.044 \\
Creatinine & 1.39 & 0.63 & 1.32 & 0.28 & -0.484 & 0.630 \\
NA & 135.95 & 4.37 & 138.45 & 3.09 & 2.366 & 0.020 \\
K & 4.01 & 0.59 & 3.88 & 0.34 & -0.887 & 0.378 \\
Hb\% & 11.19 & 0.74 & 12.33 & 1.46 & 4.550 & $<0.001$ \\
Platelet & 203.75 & 42.98 & 354.85 & 50.58 & 13.020 & $<0.001$ \\
\hline
\end{tabular}


4) Echocardiography data in patients and control groups: LVESD was higher in patients group than in control group with significant difference ( $\mathrm{P}$-value $=$ 0.001 ) and $\mathrm{EF} \%$ was lower in patients group than in control group with highly significant difference $(\mathrm{P}$-value $<0.001)$ while LVEDD revealed no significant difference $(\mathrm{P}$-value $=0.878)($ Table 3$)$.

Table 3. Echocardiography data in patients and control groups.

\begin{tabular}{ccccccc}
\hline & \multicolumn{2}{c}{$\begin{array}{c}\text { Patients group } \\
(\text { No. }=300)\end{array}$} & \multicolumn{2}{c}{$\begin{array}{c}\text { Control group } \\
(\text { No. }=20)\end{array}$} & \multicolumn{2}{c}{ Independent t-test } \\
\cline { 2 - 7 } & Mean & SD & Mean & SD & t & P-value \\
\hline LVEDD & 5.16 & 0.55 & 5.19 & 0.37 & 0.154 & 0.878 \\
LVESD & 4.06 & 0.41 & 3.72 & 0.28 & -3.430 & 0.001 \\
EF\% & 59.08 & 4.07 & 65.15 & 4.39 & 5.658 & $<0.001$ \\
\hline
\end{tabular}

E/A ratio in the patients' group and control group: E/A ratio was reversed higher in the patient group (40.0\%) than in control group with significant difference $(\mathrm{P}$-value $=0.001)($ Table 4 and Figure 1$)$.

Table 4. E/A ratio in the patient's group and control group.

\begin{tabular}{ccccccc}
\hline \multirow{2}{*}{ E/A Ratio } & \multicolumn{2}{c}{$\begin{array}{c}\text { Patients group } \\
(\text { No. }=300)\end{array}$} & \multicolumn{2}{c}{$\begin{array}{c}\text { Control group } \\
(\text { No. }=20)\end{array}$} & \multicolumn{2}{c}{ Chi-square test } \\
\cline { 2 - 6 } & No. & $\%$ & No. & $\%$ & $X^{2}$ & P-value \\
\hline Normal & 180 & $60.0 \%$ & 20 & $100.0 \%$ & & 0.001 \\
Reversed & 120 & $40.0 \%$ & 0 & $0.0 \%$ & 11.429 & \\
\hline
\end{tabular}

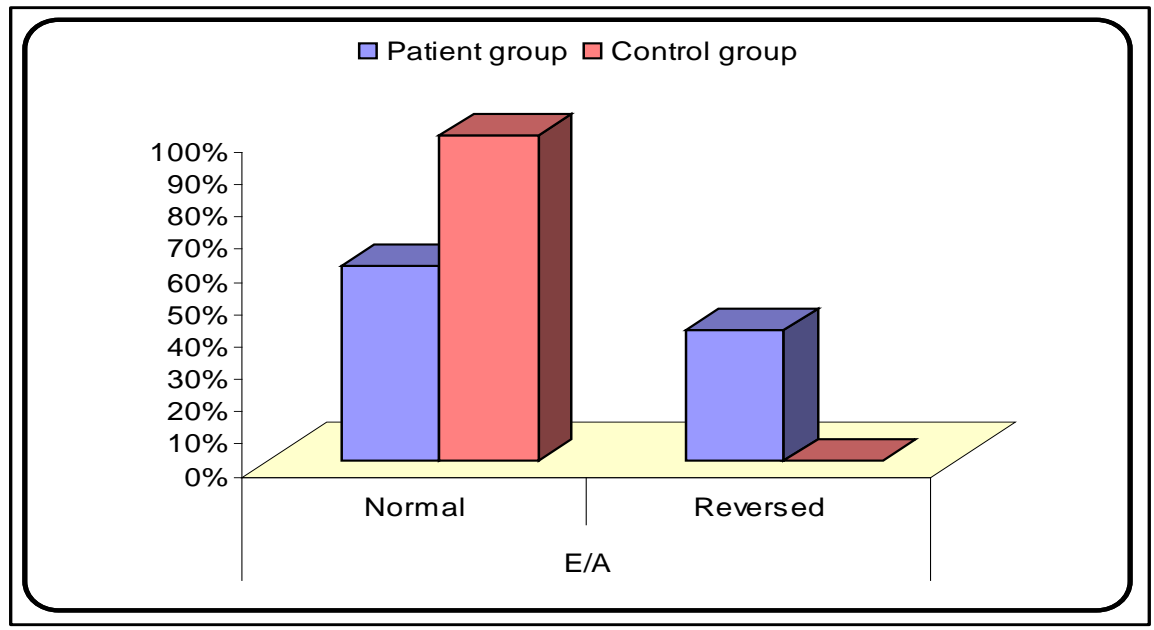

Figure 1. Comparison between patients and control group as regard E/A ratio.

\section{C) Comparison between group A and group B as regard echocardiogra-} phy data

1) Correlation between group $A$ and group $B$ as regard (LVEDD-LVESDEF\%) (Table 5). 
Table 5. There was no significant difference between group A and B as regards LVEDD, LVESD, and EF\% (P-value $=0.910-0.898-0.145)$.

\begin{tabular}{ccccccc}
\hline & \multicolumn{2}{c}{ Group A } & \multicolumn{2}{c}{$\begin{array}{c}\text { Group B } \\
150\end{array}$} & \multicolumn{2}{c}{ Independent t-test } \\
\cline { 2 - 7 } & Mean & SD & Mean & SD & t & P-value \\
\hline LVEDD & 5.15 & 0.52 & 5.17 & 0.60 & 0.113 & 0.910 \\
LVESD & 4.07 & 0.44 & 4.06 & 0.40 & -0.129 & 0.898 \\
EF\% & 59.90 & 3.60 & 58.34 & 4.47 & -1.476 & 0.145 \\
\hline
\end{tabular}

There is comparison between group A and B as regards E/A (Table 6 and Figure 2).

Table 6. The reversed E/A ratio were higher in group B more than group A with a significant difference $(\mathrm{P}$-value $=0.012)$.

\begin{tabular}{ccccccc}
\hline \multirow{2}{*}{ E/A } & \multicolumn{2}{c}{ Group A } & \multicolumn{2}{c}{ Group B } & \multicolumn{2}{c}{ Chi-square test } \\
\cline { 2 - 7 } & No. & $\%$ & No. & $\%$ & $X^{2}$ & P-value \\
\hline Normal & 115 & $76.7 \%$ & 70 & $46.7 \%$ & & 0.012 \\
Reversed & 35 & $23.3 \%$ & 80 & $53.3 \%$ & & \\
\hline
\end{tabular}

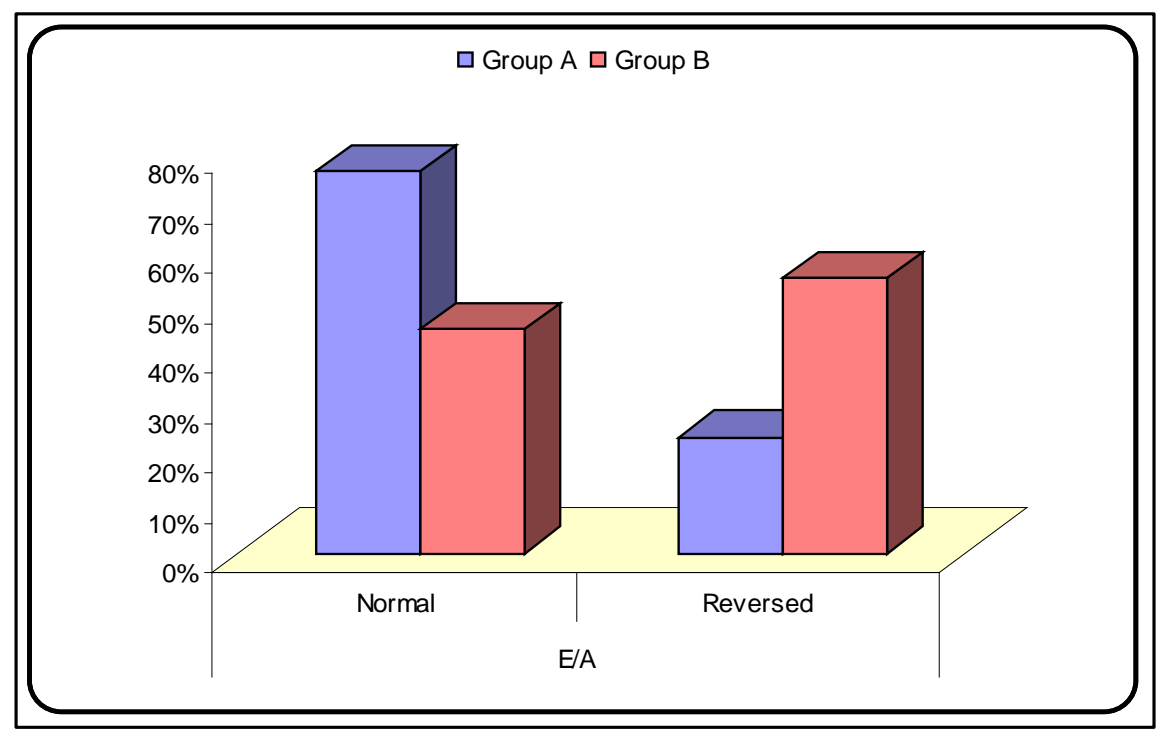

Figure 2. Comparison between group $\mathrm{A}$ and $\mathrm{B}$ as regard $\mathrm{E} / \mathrm{A}$.

D) Comparison between diastolic dysfunction and non-diastolic dysfunction in patients group (A \& B)

There is comparison between diastolic dysfunction and non-diastolic dysfunction in patients group as regard Sex distribution.

1) Age distribution: The mean age in nondiastolic dysfunction group was 51.2 while in diastolic dysfunction group was 51.33 (P-value; 0.945).

2) Sex: In patients group, sex distribution showed no significant difference 
between diastolic dysfunction and non-diastolic dysfunction groups (P-value 0.598).

3) As regard clinical data: Table 7 shows that: Ascites and encephalopathy were higher in patients who developed diastolic dysfunction than in patients who had non-diastolic dysfunction with significant difference (P-value $=0.045-0.036)$.

Table 7. Ascites and encephalopathy were higher in patients who developed diastolic dysfunction than in patients who had non-diastolic dysfunction with significant difference $(\mathrm{P}$-value $=0.045-0.036)$.

\begin{tabular}{|c|c|c|c|c|c|c|c|}
\hline \multirow{2}{*}{\multicolumn{2}{|c|}{ Patient group }} & \multicolumn{2}{|c|}{$\begin{array}{l}\text { Non diastolic dysfunction } \\
(\text { No. }=180)\end{array}$} & \multicolumn{2}{|c|}{$\begin{array}{l}\text { Diastolic dysfunction } \\
(\text { No. }=120)\end{array}$} & \multicolumn{2}{|c|}{ Chi square test } \\
\hline & & No. & $\%$ & No. & $\%$ & $X^{2}$ & $P$-value \\
\hline \multirow{2}{*}{ Ascites } & No & 115 & $63.9 \%$ & 45 & $37.7 \%$ & \multirow{2}{*}{4.029} & \multirow{2}{*}{0.045} \\
\hline & Yes & 65 & $36.1 \%$ & 75 & $62.5 \%$ & & \\
\hline \multirow{2}{*}{ Encephalopathy } & No & 165 & $91.7 \%$ & 75 & $62.5 \%$ & \multirow{2}{*}{7.656} & \multirow{2}{*}{0.036} \\
\hline & Yes & 15 & $8.3 \%$ & 25 & $37.5 \%$ & & \\
\hline
\end{tabular}

4) Comparison between diastolic dysfunction and non-diastolic dysfunction in group A (compensated liver disease).

a) As regard laboratory variables (Table 8).

Table 8. In group A (AST, ALT, Bilirubin, Urea, Creatinine, Na) were higher in patients who developed diastolic dysfunction than the patients who had nondiastolic dysfunction but with no significant differences. While (albumin, INR, and platelet) was lower in the patient who developed diastolic dysfunction than the patient who had nondiastolic dysfunction but also with no significant difference ( $\mathrm{P}$-value $=0.416-0.261-0.335)$.

\begin{tabular}{ccccccc}
\hline & $\begin{array}{c}\text { Nondiastolic dysfunction } \\
(\text { No. = 23) }\end{array}$ & \multicolumn{2}{c}{$\begin{array}{c}\text { Diastolic dysfunction } \\
\text { (No. = })\end{array}$} & \multicolumn{2}{c}{ Independent t-test } \\
\cline { 2 - 6 } & Mean & SD & Mean & SD & T & P-value \\
\hline AST & 47.48 & 20.20 & 51.17 & 17.55 & -0.408 & 0.687 \\
ALT & 53.00 & 16.21 & 58.00 & 14.79 & -0.728 & 0.473 \\
Albumin & 3.06 & 0.19 & 3.00 & 0.15 & 0.825 & 0.416 \\
INR & 1.96 & 0.17 & 1.86 & 0.30 & 1.146 & 0.261 \\
Bilirubin & 1.69 & 0.39 & 1.71 & 0.14 & -0.128 & 0.899 \\
Urea & 35.96 & 12.08 & 37.71 & 9.16 & -0.354 & 0.726 \\
Creatinine & 1.35 & 0.54 & 1.87 & 1.40 & -1.490 & 0.147 \\
Na & 136.04 & 4.35 & 136.86 & 3.76 & -0.445 & 0.660 \\
K & 3,86 & 0.44 & 3.87 & 0.69 & 0.057 & 0.955 \\
Hb\% & 10.75 & 0.76 & 11.23 & 0.72 & -1.477 & 0.151 \\
Platelet & 235.87 & 40.43 & 230.29 & 31.14 & 0.335 & 0.740 \\
\hline
\end{tabular}

b) As regard echocardiography data in group A.

There was no significant difference between diastolic dysfunction and nondiastolic dysfunction in group A as regard LVESD (3.9 vs. 4.3), LVEDD (5.1 vs. 5.06) and EF\% (60.09 vs. 59.2) (Table 9). 
Table 9. There was no significant difference between diastolic dysfunction and non-diastolic dysfunction in group A as regard LVESD, LVEDD, and EF\%.

\begin{tabular}{ccccccc}
\hline & \multicolumn{2}{c}{$\begin{array}{c}\text { No diastolic dysfunction } \\
(\text { No. = 115) }\end{array}$} & \multicolumn{2}{c}{$\begin{array}{c}\text { Diastolic dysfunction } \\
(\text { No. = 35) }\end{array}$} & \multicolumn{2}{c}{ Independent t-test } \\
\cline { 2 - 7 } & Mean & SD & Mean & SD & t & P-value \\
\hline LVEDD & 5.18 & 0.49 & 5.06 & 0.65 & 0.524 & 0.605 \\
LVESD & 3.99 & 0.29 & 4.33 & 0.71 & -1.843 & 0.076 \\
EF\% & 60.09 & 4.06 & 59.29 & 1.25 & 0.51 & 0.614 \\
\hline
\end{tabular}

c) As regards E/A ratio in group A: Reversed E/A was higher in patients who developed diastolic dysfunction than the patient who had nondiastolic dysfunction with highly significant difference (P-value $<0.001)$ (Table 10$)$.

Table 10. Reversed E/A was higher in patients who developed diastolic dysfunction than the patient who had non diastolic dysfunction with highly significant difference (P-value $<0.001)$.

\begin{tabular}{ccccccc}
\hline \multirow{2}{*}{ E/A } & \multicolumn{2}{c}{$\begin{array}{c}\text { Non D.D } \\
(\text { No. }=115)\end{array}$} & \multicolumn{2}{c}{$\begin{array}{c}\text { D.D } \\
(\text { No. }=35)\end{array}$} & \multicolumn{2}{c}{ Chi-square test } \\
\cline { 2 - 7 } & No. & $\%$ & No. & $\%$ & $X^{2}$ & P-value \\
\hline Normal & 115 & $100.0 \%$ & 0 & $0.0 \%$ & & \\
Reversed & 0 & $0.0 \%$ & 35 & $100.0 \%$ & & \\
\hline
\end{tabular}

5) Comparison between diastolic dysfunction and non-diastolic dysfunction in group B:

a) As regard laboratory data in group B. There was no significant difference between diastolic and nondiastolic dysfunction as regard labs (Table 11).

Table 11. Laboratory findings in diastolic dysfunction and nondiastolic dysfunction in group B.

\begin{tabular}{ccccccc}
\hline & \multicolumn{2}{c}{$\begin{array}{c}\text { No diastolic dysfunction } \\
(\text { No. }=65)\end{array}$} & \multicolumn{2}{c}{$\begin{array}{c}\text { Diastolic dysfunction } \\
(\text { No. }=85)\end{array}$} & \multicolumn{2}{c}{ Independent t-test } \\
\cline { 2 - 6 } & Mean & SD & Mean & SD & t & P-value \\
\hline AST & 59.62 & 21.37 & 52.59 & 19.40 & -0.941 & 0.355 \\
ALT & 73.31 & 17.37 & 66.69 & 19.78 & -0.946 & 0.353 \\
Albumin & 2.28 & 0.44 & 2.05 & 0.51 & -1.345 & 0.190 \\
INR & 2.02 & 0.42 & 1.76 & 0.44 & -1.684 & 0.103 \\
Bilirubin & 3.35 & 2.52 & 3.22 & 1.99 & -0.164 & 0.871 \\
Urea & 38.31 & 15.38 & 46.19 & 33.82 & 0.778 & 0.443 \\
Creatinine & 1.36 & 0.39 & 1.28 & 0.32 & -0.655 & 0.518 \\
Na & 136.31 & 5.02 & 135.18 & 4.35 & -0.660 & 0.514 \\
K & 4.06 & 0.58 & 4.23 & 0.69 & 0.723 & 0.476 \\
Hb\% & 11.62 & 0.57 & 11.42 & 0.57 & -0.965 & 0.343 \\
Platelet & 168.23 & 16.67 & 176.53 & 19.69 & 1.221 & 0.232 \\
\hline
\end{tabular}


b) As regard to echocardiography data in group $\mathrm{B}$.

The (LVEDD-LVESD-EF\%) show no significant difference between diastolic dysfunction and non-diastolic dysfunction (P-value 0.186-0.641-0.303) in group B.

Comparison between diastolic dysfunction and non-diastolic dysfunction as regard $\mathrm{E} / \mathrm{A}$ ratio in group $\mathrm{B}$.

Reversed E/A was higher in patients who developed diastolic dysfunction than the patient who had nondiastolic dysfunction with highly significant difference (P-value < 0.001).

6) Comparison between diastolic dysfunction and non-diastolic dysfunction in group $\mathrm{A}$, group $\mathrm{B}$, and control.

a) Comparison between group A \&control as regard diastolic dysfunction (Table 12).

Table 12. There was diastolic dysfunction in group A more than control group with significant difference $(\mathrm{P}$-value $=0.019)$.

\begin{tabular}{ccccccc}
\hline \multirow{2}{*}{ Groups } & \multicolumn{2}{c}{$\begin{array}{c}\text { No diastolic dysfunction } \\
(\text { No. = 115) }\end{array}$} & \multicolumn{2}{c}{$\begin{array}{c}\text { Diastolic dysfunction } \\
(\text { No. = 35) }\end{array}$} & \multicolumn{2}{c}{ Chi-square test } \\
\cline { 2 - 7 } & No. & $\%$ & No. & $\%$ & $X^{2}$ & P-value \\
\hline Group A & 115 & $76.6 \%$ & 35 & $23.4 . \%$ & & \\
Control group & 20 & $100 \%$ & 0 & $0.0 \%$ & & \\
\hline
\end{tabular}

b) Comparison between group B \& control as regard diastolic dysfunction, Table 13.

Table 13. There was diastolic dysfunction in group B more than in the control group with a highly significant difference $(\mathrm{P}$-value $<0.001)$.

\begin{tabular}{ccccccc}
\hline \multirow{2}{*}{ Groups } & \multicolumn{2}{c}{ No diastolic dysfunction } & \multicolumn{2}{c}{ Diastolic dysfunction } & \multicolumn{2}{c}{ Chi-square test } \\
\cline { 2 - 7 } & No. & $\%$ & No. & $\%$ & $\mathrm{X}^{2}$ & P-value \\
\hline Group B & 65 & $43.3 \%$ & 85 & $56.7 \%$ & \multirow{2}{*}{17.172} & $<0.001$ \\
Control group & 20 & $100 \%$ & 0 & $0.0 \%$ & & \\
\hline
\end{tabular}

Comparison between the study groups (patients \&control) as regard Diastolic dysfunction (Figure 3). There was diastolic dysfunction in patients group more than in the control group with significant difference (P-value 0.001).

c) Comparison between group A group B control as regard diastolic dysfunction. There were highly significant differences $(\mathrm{P}$-value $<0.001)$ as regard diastolic dysfunction as group B resemble (70.8\%) while group A (29.2\%) and control group (0.0\%) (Table 14 and Figure 4$)$.

\section{Discussion}

Liver cirrhosis is associated with a wide range of cardiovascular abnormalities, including hyperdynamic circulation, cirrhotic cardiomyopathy, and pulmonary vascular abnormalities. Accumulating evidence suggests that cirrhosis related 


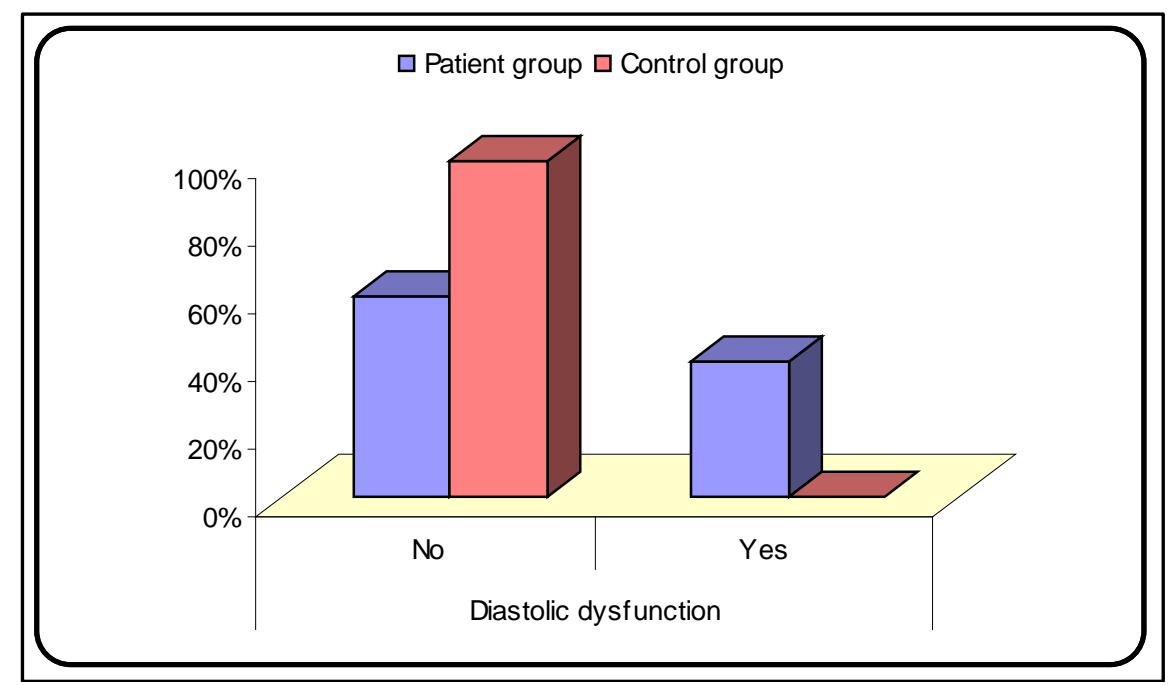

Figure 3. Comparison between the study groups as regard Diastolic dysfunction.

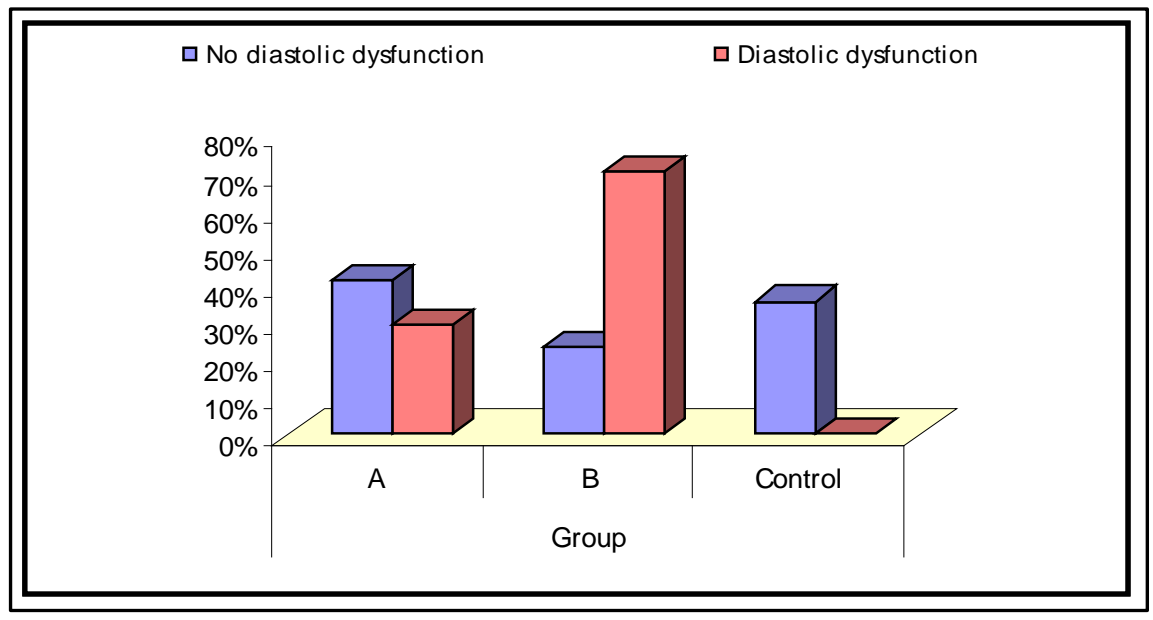

Figure 4. Comparison between group A \& group B \& control as regard diastolic dysfunction.

Table 14. Comparison between group A \& group B \& control as regard diastolic dysfunction (Figure 4).

\begin{tabular}{ccccccc}
\hline \multirow{2}{*}{ Groups } & \multicolumn{2}{c}{$\begin{array}{c}\text { No diastolic dysfunction } \\
(\text { No. }=180)\end{array}$} & \multicolumn{2}{c}{$\begin{array}{c}\text { Diastolic dysfunction } \\
(\text { No. = 120) }\end{array}$} & \multicolumn{2}{c}{ Chi-square test } \\
\cline { 2 - 7 } & No. & $\%$ & No. & $\%$ & $X^{2}$ & P-value \\
\hline A & 115 & $41.1 \%$ & 35 & $29.2 \%$ & & \\
B & 65 & $23.2 \%$ & 85 & $70.8 \%$ & & $<0.001$ \\
\hline
\end{tabular}

cardiovascular abnormalities play a significant role in the pathogenesis of multiple life-threatening complications, including hepatorenal syndrome, ascites, spontaneous bacterial peritonitis, gastroesophageal varices, and hepatopulmonary syndrome. Treatment targeting the circulatory dysfunction in these patients may improve short-term prognosis while awaiting liver transplantation [4]. 
Cirrhotic cardiomyopathy is a clinical syndrome in patients with liver cirrhosis characterized by an abnormal and blunted response to physiologic, pathologic, or pharmacologic stress but typical to increased cardiac output and contractility at rest. As many as $50 \%$ of cirrhotic patients undergoing liver transplantation show signs of cardiac dysfunction, and $7 \%$ to $21 \%$ of deaths after orthotropic liver transplantation result from apparent heart failure [5].

The present study was done to investigate the diastolic function changes prevalence in viral related liver cirrhosis. It was performed on 300 patients with HCV related liver cirrhosis and twenty control. The patients' group were subdivided according to compensation into two groups, group A was 150 patients with Compensated Liver cirrhosis disease (child A) 80 of them (53.3\%) were females, and $70(46.7 \%)$ males. The age ranged between 38 - 60 years with a mean age $(51.52 \pm 6.06)$. And group B was 150 patient with Decompensate Liver cirrhosis disease (child B $+\mathrm{C}$ ), 70 of them (46.7\%) were females and $80(53.3 \%)$ males. The age ranged between 39 - 60 years with mean age $(50.37 \pm 6.15)$, and a control group of 20 persons 11 (55\%) females and 9 (45\%) males. The age ranged between 40 - 55 years with mean age $(48.8 \pm 3.27)$.

In this study, we found significant abnormalities in left ventricular diastolic function in patients with liver cirrhosis in the form of a reversed E/A ratio; this is in agreement with [6]. Also, these results are in agreement with Mohamed et al. [6], who found a significant decrease in E/A ratio in cirrhotic patients. This indicates a high frequency of left ventricular diastolic dysfunction in patients with cirrhotic liver disease.

In this study decreased $\mathrm{E} / \mathrm{A}$ ratio as an indicator for diastolic dysfunction was found in 120 (40\%) patients while patients had standard E/A ratio was 180 $(60 \%)$, this percentage is lower than that found by Fu-Rong Sun et al. [7], who found that nearly $50 \%$ of patients showed an $\mathrm{E} / \mathrm{A}$ ratio $\leq 1$. The population of Fu-Rong study was heterogonous (including HBV, HCV, and alcoholic cirrhosis).

In this study the Laboratory data in group A (AST, ALT, Bilirubin, Urea, Creatinine, $\mathrm{Na}, \mathrm{Hb} \%$ ) were higher in patients who developed diastolic dysfunction than in the patients who had non diastolic dysfunction but with no significant differences. This means that the diastolic function properties of the left ventricle become more pronounced in patients with more progressive deterioration of liver functions. While Albumin was lower in patients who developed diastolic dysfunction than the patients who had non diastolic dysfunction but with no significant differences This finding may draw attention to the role of albumin deficiency in the pathophysiology of heart failure, especially considering that previous studies had presumed that normal levels of serum albumin might have direct protective effects such as anti-apoptotic and antioxidant activity [8].

While in group B (decompensate liver disease) there were no significant differences between diastolic dysfunction and non-diastolic dysfunction regarding all laboratory Variables as (ALT-AST-albumin-INR-bilirubin-Na-......). 
In this study: ascites in patients group was found to be higher in the patients who developed diastolic dysfunction than the patient with nondiastolic dysfunction with significant difference $(\mathrm{P}$-value $=0.045)$. This is in agreement with Baik et al. [9], who reported that diastolic dysfunction appears to be more common in cirrhotic patients with ascites than those without ascites and that some degree of diastolic dysfunction is present in virtually every patient with cirrhosis which manifests as a stiff, noncompliant ventricle and that impaired passive and active filling of the left ventricle in early and middle-late diastole, respectively, lead to an inability to adequately increase stroke volume in response to stimuli and in contrast to systolic dysfunction, a significant stimulus may not be required to detect diastolic dysfunction and that echocardiography may reveal abnormal diastolic function even at rest. Also agreement with Møller and Henriksen [10], who showed that $\mathrm{A}$-wave and $\mathrm{E}$-wave velocities are much increased, and the E/A-ratio is reversed in cirrhotic patients, especially in those with ascites, and that the presence of a subclinical myocardial disease with diastolic dysfunction, which, in ascitic patients, is improved after paracentesis and aggravated after TIPS.

All patients in this study had LVESD higher than the control group, with a significant difference between control and cirrhotic patients ( $\mathrm{P}$-value $=0.001)$. These results are in contrast to findings of (Soyoral et al., [11]), who found no changes in ventricular dimensions and explained this by the facilitation of left ventricle performance because of decreased peripheral vascular resistance.

Comparison between patients based upon the severity of the disease revealed significant difference among the two groups regarding the prevalence of diastolic dysfunction $(\mathrm{P}=0.012)$ which was found to be significantly increased with increasing the severity of the liver disease. This result in agreement with the results of Trevisani et al. [12], and Baik et al. [9], who reported that the degree of circulatory hyperkinesis and structural changes correlate with the degree of severity of liver cirrhosis. Concordant with Ho and Youshida, [13], who reported that the diastolic dysfunction was significantly present in all cirrhotic patients, but the severity was increased with the increased severity of the chronic liver disease. In contrast to systolic dysfunction, diastolic dysfunction appears to be more prevalent, and some degree of diastolic dysfunction is present in virtually every patient with cirrhosis.

By increasing the severity of cirrhosis from Child A to Child C, normal diastolic function was decreased, and diastolic dysfunction was increased. In contrast to our study results, Genovesi et al. showed that echocardiographic findings have no significant differences between groups [14].

Finally, Cardiac dysfunction is a common complication of advanced cirrhosis that can make a variety of disturbances, especially diastolic dysfunction.

Because of the high prevalence of diastolic dysfunction in cirrhotic patients and the risk of decompensation following invasive procedures, it could be suggested that all patients would be screened routinely by echocardiography before invasive procedures. 


\section{Conclusion}

Liver cirrhosis is associated with echocardiographic changes in the form of diastolic dysfunction, and these changes are evident in patients with decompensated liver cirrhosis more than those with compensated liver cirrhosis, and these changes do not depend on variations in the left ventricular geometry measurements. These structural hemodynamic abnormalities of cardiac functions in liver cirrhosis are termed as cirrhotic cardiomyopathy. However, these changes may follow a silent course, hence making an early diagnosis and appropriate management difficult. Full-blown cirrhotic diastolic dysfunction carries some risk of heart failure if not appropriately treated. Furthermore, cirrhotic diastolic dysfunction may be a mechanism involved in the pathogenesis of hepatorenal syndrome, ascites, and variceal bleeding, and it can be a critical factor preventing liver transplantation. Thus, prompt recognition and appropriate management of diastolic dysfunction are integral parts of the management for patients with end-stage liver disease awaiting liver transplantation.

\section{Recommendations}

1) Echocardiography follows up of patients with cirrhosis is highly recommended because the present study and many previous studies reported many significant Echocardiographic changes in these patients underlying the term cirrhotic cardiomyopathy.

2) Cirrhotic patients who are preparing for liver transplantation or will undergo procedures like TIPS or surgical shunts or any surgery should have Echocardiography and cardiac assessment fearing that inappropriate management of fluid balance and fluid overload would precipitate overt heart failure in the already latently affected heart in cirrhotics.

3) Decrease E/A ratio are a prognostic marker for liver cirrhosis worsening and should be regarded in this aspect in addition to other scoring systems.

4) Awareness about the cardiac complication of cirrhosis should be increased, especially among surgeons and anesthesia doctors dealing with Cirrhotic patients.

5) Cardiologists should be involved in the care of Cirrhotic patients, especially in preoperative periods for any surgery and in the whole per transplantation period in case of liver transplantation.

\section{Conflicts of Interest}

The authors declare no conflicts of interest regarding the publication of this paper.

\section{References}

[1] Ahmed, A.M., Hassan, M.S., Abd-Elsayed, A., et al. (2011) Insulin Resistance, Steatosis, and Fibrosis in Egyptian Patients with Chronic Hepatitis C Virus Infection. Saudi Journal of Gastroenterology, 17, 245-251. https://doi.org/10.4103/1319-3767.82578 
[2] Mohammad Nasr, F., Metwaly, A., khalik, A.A. and Darwish, H. (2015) Cardiac Dysfunction in Liver Cirrhosis: A Tissue Doppler Imaging Study from Egypt. Electron Physician, 7, 1135-1143.

[3] Jessup, M., Abraham, W.T., Casey, D.E., et al. (2009) Focused Update: ACCF/AHA Guidelines for the Diagnosis and Management of Heart Failure in Adults: A Report of the American College of Cardiology Foundation/American Heart Association Task Force on Practice Guidelines Developed in Collaboration with the International Society for Heart and Lung Transplantation. Journal of the American College of Cardiology, 53, 1343-1382. https://doi.org/10.1016/j.jacc.2008.11.009

[4] Finucci, G., Desideri, A., Sacerdoti, D., et al. (1996) Left Ventricular Diastolic Function in Cirrhosis. Scandinavian Journal of Gastroenterology, 31, 279-284. https://doi.org/10.3109/00365529609004879

[5] Al-Hamoudi, W.K. (2010) Cardiovascular Changes in Cirrhosis: Pathogenesis and Clinical Implications. Scandinavian Journal of Gastroenterology, 16, 145-153. https://doi.org/10.4103/1319-3767.65181

[6] Mohamed, A.D. and Aghnaya, A.D. (2012) Hepatitis C Virus in Arab World: A State of Concern. Scientific World Journal, 2012, Article ID: 719494. https://doi.org/10.1100/2012/719494

[7] Fullwood, D. (2012) Portal Hypertension and Varices in Patients with Liver Cirrhosis. Nursing Standard, 26, 52-57.

[8] Braunwald, E. (2008) Evaluation of the Patient; Evaluation of Systolic and Diastolic Function. In: Echocardiography, 8th Edition.

[9] Baik, S.K., Fouad, T.R. and Lee, S.S. (2007) Cirrhotic Cardiomyopathy. Orphanet Journal of Rare Diseases, 27, 2-15. https://doi.org/10.1186/1750-1172-2-15

[10] Moller, S. and Henriksen, J.H. (2010) Cirrhotic Cardiomyopathy. Journal of Hepatology, 53, 179-190.

[11] Soyoral, Y., Süner, A., Kıdır, V., et al. (2004) The Effect of Viral Cirrhosis on Cardiac Ventricular Function. European Journal of General Medicine, 1, 15-18.

[12] Trevisani, F., Merli, M., Savelli, F., et al. (2003) QT Interval in Patients with Non-Cirrhotic Portal Hypertension and Cirrhotic Patients Treated with a Transjugular Intrahepatic Porto-Systemic Shunt. Journal of Hepatology, 38, 461-467. https://doi.org/10.1016/S0168-8278(03)00057-6

[13] Ho, J.K. and Youshida, E. (2006) The Extrahepatic Consequences of Cirrhosis. Medscape General Medicine, 8, 59.

[14] Genovesi, S., Prata Pizzala, D.M., Pozzi, M., et al. (2009) QT Interval Prolongation and Decreased Heart Rate Variability in Cirrhotic Patients: Relevance of Hepatic Venous Pressure Gradient and Serum Calcium. Clinical Science, 116, 851-859. https://doi.org/10.1042/CS20080325 